\title{
Acoustic levitation in mid-air: Recent advances, challenges, and future perspectives
}

Cite as: Appl. Phys. Lett. 116, 250501 (2020); https://doi.org/10.1063/5.0012660

Submitted: 04 May 2020 . Accepted: 09 June 2020 . Published Online: 22 June 2020

Marco A. B. Andrade, (D) Asier Marzo, and Julio C. Adamowski

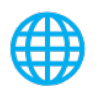

\section{ARTICLES YOU MAY BE INTERESTED IN}

TinyLev: A multi-emitter single-axis acoustic levitator

Review of Scientific Instruments 88, 085105 (2017); https://doi.org/10.1063/1.4989995

Contactless pick-and-place of millimetric objects using inverted near-field acoustic levitation Applied Physics Letters 116, 054104 (2020); https://doi.org/10.1063/1.5138598

Microwave applications of photonic topological insulators

Applied Physics Letters 116, 250502 (2020); https://doi.org/10.1063/5.0008046

\section{Challenge us.}

What are your needs for periodic signal detection?

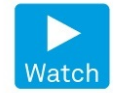

- Zurich

Instruments

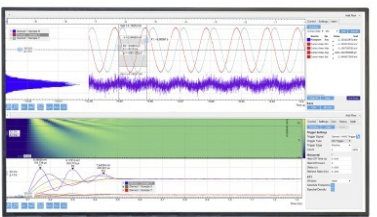

( 


\title{
Acoustic levitation in mid-air: Recent advances, challenges, and future perspectives
}

\author{
Cite as: Appl. Phys. Lett. 116, 250501 (2020); doi: 10.1063/5.0012660 \\ Submitted: 4 May 2020 - Accepted: 9 June 2020 . \\ Published Online: 22 June 2020

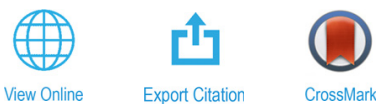

Marco A. B. Andrade, ${ }^{1, a)}$ (D) Asier Marzo, ${ }^{2}$ (D) and Julio C. Adamowski ${ }^{3}$

\author{
AFFILIATIONS \\ 'Instituto de Física, Universidade de São Paulo, São Paulo 05508-090, Brazil \\ ${ }^{2}$ UpnaLab, Universidad Pública de Navarra, Pamplona 31006, Navarra, Spain \\ ${ }^{3}$ Escola Politécnica, Universidade de São Paulo, São Paulo 05508-030, Brazil \\ ${ }^{a)}$ Author to whom correspondence should be addressed: marcobrizzotti@gmail.com
}

\begin{abstract}
Mid-air acoustic levitation is becoming a powerful tool to suspend and manipulate millimetric objects. Because of its unique characteristics, acoustic levitation is suitable to trap a wide variety of materials such as liquids, solids, soap bubbles, and even living creatures. Acoustic levitation can also be combined with noncontact measurement systems, allowing contactless analysis and characterization of levitating samples. In this article, we review some of the advances that have been made over the last decade. We also present the technical challenges that must be overcome in order to extend the capability of current acoustic levitation devices and, finally, we point out future directions for acoustic levitation.
\end{abstract}

Published under license by AIP Publishing. https://doi.org/10.1063/5.0012660

Acoustic levitation $^{1,2}$ uses the acoustic radiation force ${ }^{3}$ exerted by sound waves to suspend objects in mid-air. Acoustic levitation is not only an interesting physical phenomenon but also a promising tool for multiple disciplines such as biology, chemistry, or pharmacy. The unique features of acoustic levitation also have great potential for performing some tasks in industry such as the contactless manipulation of fragile objects or processing small volumes of liquids. In this article, we first highlight several breakthroughs that have been achieved over the last decade. Then, we describe some of the technical challenges that must be overcome to untap the full potential of acoustic levitation. Finally, we present emerging applications for acoustic levitation, which are becoming more and more established. The physics behind acoustic levitation may also be employed for trapping, sorting, and manipulating microparticles and cells inside a liquid medium, ${ }^{4-6}$ but in this article, we focus our attention on mid-air acoustic levitation.

The first experiments with acoustic levitation date back to 1933, when Bücks and Müller ${ }^{7}$ reported the acoustic suspension of ethanol droplets at the pressure nodes of a standing wave generated between a vibrating rod and a reflecting wall. Although acoustic levitation was demonstrated almost a century ago, most significant advances have been made in recent years. From the early 1930s to the first decade of this century, the usual method for acoustically levitating objects has remained mainly the same. Most of the experiments trapped a small object (relative to the acoustic wavelength) in a pressure node of a standing wave, which was generated either using a single-axis levitator ${ }^{8-11}$ composed of an emitter and an opposing reflector [Fig. 1(a)] or inside a closed cavity. ${ }^{12}$ Most standing wave levitators operate in the ultrasonic range (with a typical frequency between 20 and $100 \mathrm{kHz}$ ) and they are suitable for trapping objects ranging from hundreds of micrometers to few millimeters although acoustic levitation may also be employed for levitating smaller or larger objects.

Nowadays, acoustic levitation systems are able not only to trap but also to rotate and translate the particles in three dimensions. Furthermore, acoustic levitation is no longer restricted to the Rayleigh regime (i.e., particles much smaller than the acoustic wavelength), and distinct approaches are available to trap in the Mie regime (i.e., particle size comparable to the wavelength).

Over the last decade, we have seen vast advances in acoustic levitation. Starting in 2010, Koyama and Nakamura showed a system based on linear transporters ${ }^{14}$ and contactless rotary junctions. ${ }^{15}$ In 2013, Foresti et al. ${ }^{13}$ demonstrated the contactless transportation and merging of multiple drops in two dimensions using an array of highpower Langevin transducers and an opposing reflector. This levitation concept, illustrated in Fig. 1(b), can be of great interest for performing chemical reactions between drops and in DNA transfection of cells. ${ }^{16}$ One of the most popular developments of the last decade was achieved by Ochiai et al., ${ }^{17-19}$ who developed an acoustic levitation system consisting of four orthogonal arrays made of low-power ultrasonic 
(a)

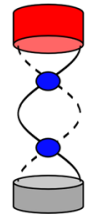

(b)

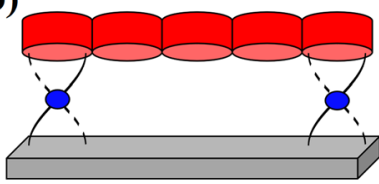

(c)

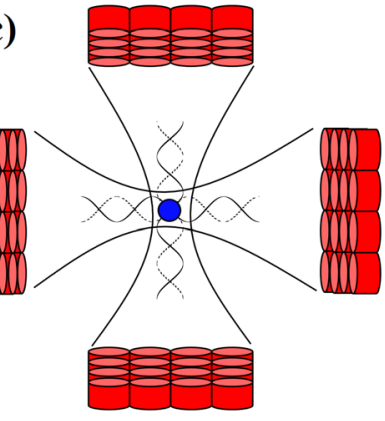

(d)

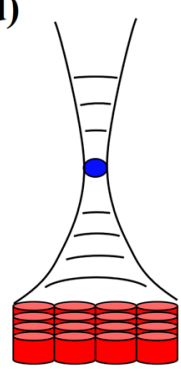

Qemitter
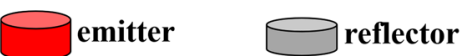

Oobject

FIG. 1. Schematic of different types of acoustic levitators. (a) Single-axis acoustic levitator. (b) Two-dimensional manipulation of multiple drops using an array of Langevin-type transducers and an opposing reflector. (c) Three-dimensional manipulation of levitated particles using four orthogonal arrays of ultrasonic emitters. (d) Single-beam trapping.

emitters that were able to control the position of levitated objects in three dimensions [Fig. 1(c)]. This work also marked the transition from the use of high-power Langevin transducers to arrays made of dozens of small low-power ultrasonic emitters (see the review article by Morris et al. $^{20}$ ).

Holographic techniques, like the ones used in optical trapping, ${ }^{21-23}$ have also been adapted to acoustic levitation. The phases at each point of the emitter plane can be set to generate intricate acoustic fields that trap particles at the target location, using either phased-arrays ${ }^{24-26}$ with dozens of emitters [Fig. 2(a)] or passive modulators $^{27-30}$ [Fig. 2(b)]. The phases can be set with previously known patterns or obtained using optimization techniques. ${ }^{24}$ Using the holographic methods, an object can be trapped using just a singlesided array, as shown in Fig. 1(d), and it is also possible to trap multiple particles and move them individually. ${ }^{26}$ When phased-arrays are employed, the levitated objects can be moved in real time; however, the reduced number of emitters leads to limitations in spatial resolution and, thus, in the ability to produce complex acoustic fields. ${ }^{27,31}$ On the other hand, passive structures can create more intricate fields, ${ }^{27-30}$ but they cannot dynamically change the field to move the particles. $^{27,29}$

Objects larger than the acoustic wavelength have been levitated during the past decade. This can be achieved either by generating a standing wave field between the emitter and the object ${ }^{32-34}$ or with special vortices of high apperture. ${ }^{25,35}$ Figure 2 (c) shows the acoustic levitation of a sphere of $30 \mathrm{~mm}$ diameter ( 3.5 wavelengths at $40 \mathrm{kHz}$ ) using an array of 996 low-power transducers.

Despite the recent advancements in mid-air acoustic levitation, there are multiple technical challenges still to overcome. For example,

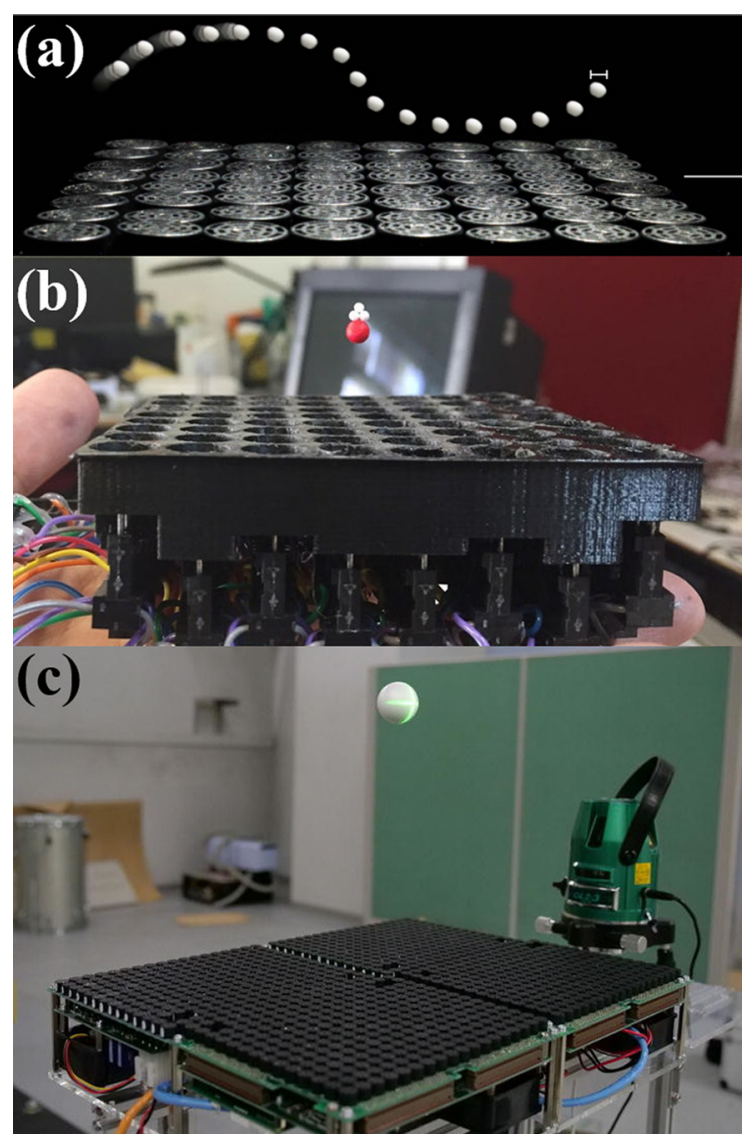

FIG. 2. (a) Dynamic manipulation of a $2 \mathrm{~mm}$ diameter Styrofoam particle using a single-sided array. [Reprinted with permission from Marzo et al., Nat. Commun. 6, 8661 (2015). ${ }^{24}$ Copyright 2015 Authors, licensed under a CC BY 4.0.] (b) Levitation with a field modulated by a 3d-printed passive structure. [Reprinted with permission from Marzo et al., Appl. Phys. Lett. 110, 014102 (2017). ${ }^{30}$ Copyright 2017 AIP Publishing.] (c) Levitation of a $30-\mathrm{mm}$ diameter sphere ( 3.5 wavelengths at $40 \mathrm{kHz}$ ) using an array of 996 transducers. [Reprinted with permission from Inoue et al., J. Acoust. Soc. Am. 145, 328 (2019). ${ }^{25}$ Copyright 2019 Acoustic Society of America.]

increasing the manipulation speed of the trapped particles requires more insight into the dynamics of acoustically levitated particles. The trap force is usually modeled as a damped harmonic oscillator with a restoring force based on an ideal spring, ${ }^{36-38}$ but at high speeds, the trapped object may present large displacements with respect to the position of minimum force potential. In this condition, models based on a linear trapping stiffness are no longer valid and the acoustic restoring force must be described by nonlinear stiffness models ${ }^{39}$ to capture the softening of stiffness and period-doubling bifurcations that occur in the particle dynamics. Speeds of up to $8.75 \mathrm{~m} / \mathrm{s}$ have been shown ${ }^{40}$ on lightweight particles; however, moving heavier particles at high speed is still not possible. Similarly, dampening undesired oscillations of trapped solid objects inside a levitator is not a trivial problem, ${ }^{41}$ and this issue becomes more acute when liquid drops are levitated. ${ }^{42}$ To obtain a reliable apparatus usable by the wider scientific community, acoustic levitators would require automatic adjustments of their parameters (e.g., operation frequency or emission phase); for 
fast movement of the particles, a better understanding of the dynamics is needed.

To date, controlled orientation of an object in three dimensions has not been demonstrated. So far, the orientation has been controlled only in one dimension, ${ }^{13,24}$ but a full control on the orientation, such as that simulated by Helander et al., ${ }^{43}$ would enable applications in spectroscopy, photogrammetry, and contactless placement. Additionally, the orientation of spherical objects cannot be adjusted given their symmetries; it is possible to impart angular speed into levitated spheres, ${ }^{35,44,45}$ but control on static orientation is still to be demonstrated. Dynamic levitation of multiple high-density objects would open up numerous possibilities; however, the trapping strength decreases linearly with the number of traps ${ }^{26}$ and the current arrays do not have enough power to levitate multiple high-density objects simultaneously. Further improvement requires the development of arrays with more powerful and frequency-stable emitters. The acoustic levitation and manipulation of multiple objects also require better knowledge of the interparticle radiation forces ${ }^{46,47}$ (also called secondary force) caused by the scattered wave from other trapped objects.

Further advancements also require better models and theoretical understanding of the forces acting on the levitated objects. With few exceptions, most models employ the Gor'kov ${ }^{48}$ equation to simulate the acoustic radiation force acting on the levitated objects, which is only valid for spherical objects much smaller than the acoustic wavelength. Furthermore, relevant physical phenomena, such as acoustic streaming, ${ }^{49-51}$ acoustic viscous torques, ${ }^{52}$ and harmonic generation, ${ }^{53,54}$ are ignored in most models.

Over the last few years, we have seen the emergence of multiple applications for acoustic levitation, but so far they are restricted to academic research. For instance, acoustic levitation has been used as a contactless platform for manipulating and mixing samples of living cells and DNA plasmids, ${ }^{16}$ as shown in Fig. 3(a). Acoustic levitation has also been applied to suspend samples of protein crystals, while synchrotron $\mathrm{x}$-ray diffraction is employed for characterizing its molecular structure ${ }^{55}$ [Fig. 3(b)]. It has also been demonstrated that electronic components can be manipulated without contact using inverted-near field acoustic levitation [Fig. 3(c)]. Another interesting use for acoustic levitation was to create a volumetric display with a fast-moving levitated particle [Fig. 3(d)].

Despite its increasing use in academic research, there is still no industrial application or mass-produced solution based on acoustic levitation, i.e., a "killer application" with the potential to revolutionize industrial processes. Acoustic levitation could be the base technology for a contactless liquid processing platform with the ability to programmatically mix, split, and translate droplets without contact. This platform would allow the same operations as current electrowetting systems, ${ }^{57,58}$ but given the wide range of wavelengths that acoustic systems can employ, it would be capable of operating from the micro to the milliliter scale. However, in contrast to electrowetting systems, splitting liquid or granular media is not possible yet with acoustic levitation; mixing has been achieved, ${ }^{13,59,60}$ but for a complete contactless fluidic solution, splitting is an essential operation. Mid-air acoustic levitation systems are becoming more widely available in either do-it-yourself (DIY) flavours ${ }^{61,62}$ or commercial equipment [e.g., Manipulator Model D, Neurotechnology_Ultrasound Research Group; L800, BOROSA Acoustic Levitation GmbH; Single-Axis Acoustic Levitator $\left(\mathrm{SAL}^{\mathrm{TM}}\right)$, Materials Development, Inc.]. However,

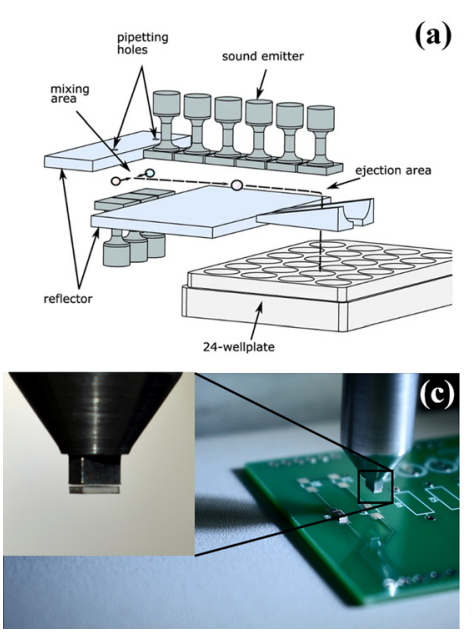

(a)

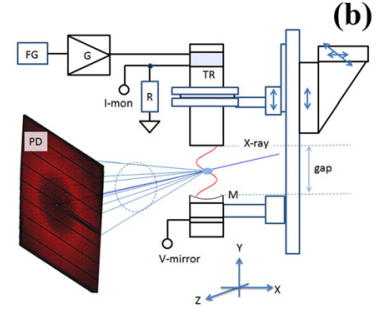

(b)

FIG. 3. Potential applications for acoustic levitation: (a) acoustic levitation method for DNA transfection of cells. [Reprinted with permission from Vasileiou et al., Sci. Rep. 6, 20023 (2016). ${ }^{16}$ Copyright 2016 Authors, licensed under a CC BY 4.0.] (b) $\mathrm{X}$-ray diffraction of protein crystals in levitated drops. [Reprinted with permission from S. Tsujino and T. Tomizaki, Sci. Rep. 6, 25558 (2016). ${ }^{55}$ Copyright 2016 Authors, licensed under CC BY 4.0.] (c) Pick-and-place of electronic components using inverted near-field acoustic levitation. [Adapted with permission from Andrade et al., Appl. Phys. Lett. 116, 054104 (2020). ${ }^{56}$ Copyright 2020 AIP Publishing.] (d) Volumetric display with a fast-moving particle and persistence of vision. [Reprinted with permission from Hirayama et al., Nature 575, 320 (2019). ${ }^{40}$ Copyright 2019 Springer Nature.]

this is not the case for trapping systems that operate with water as the propagation medium, even when many applications are only possible in water-based media.

Promising applications and future research will be derived in the next few years. Increasingly more synchrotron experiments use acoustically levitated samples to increase the sensitivity and provide unprecedented results. With the appearance of affordable and simple to use levitators, these experiments will keep raising in popularity. Another promising application is to use acoustic levitation for producing amorphous drugs with enhanced solubility. ${ }^{63,64}$ On the other hand, contactless picking and placing of fragile components can be performed by inverted-near-field acoustic levitation ${ }^{56,65}$ and replace current methods based on vacuum suckers that can scratch, shock, or cross-contaminate sensitive components. Another emergent research topic is displays made up of levitating particles acting as physical voxels or tracers: from uniform groups of particles, ${ }^{19}$ charts $^{66}$ and props ${ }^{67}$ to fast-moving particles with persistence of vision. ${ }^{40,68}$ Finally, trapping in nonhomogenous media is an open research topic, and most simulations and experiments have been conducted in open space. However, the capacity of sound to reflect and diffract could enable interesting possibilities to trap around obstacles or in porous media.

In summary, we have presented some of the recent advances in acoustic levitation. We also point out some technical challenges that must be overcome in order to extend the manipulation capabilities of current levitation devices. So far, acoustic levitation has been restricted to applications in academic research, but we expect that industrial applications will emerge and be applied in the following years. In the near future, the current levitation devices may evolve to the point that 
they would be able to manipulate millimetric objects with the same dexterity as industrial robots, but without any contact between the robot and the object to be manipulated.

This work was supported by the São Paulo Research Foundation-FAPESP (Grant No. 2017/27078-0) and by the Government of Navarre through Fondo Europeo de Desarrollo Regional (FEDER) Project No. 0011-1365-2019-000086.

\section{DATA AVAILABILITY}

Data sharing is not applicable to this article as no new data were created or analyzed in this study.

\section{REFERENCES}

${ }^{\mathbf{1}}$ E. H. Brandt, Nature 413, 474 (2001).

${ }^{2}$ M. A. B. Andrade, N. Pérez, and J. C. Adamowski, Braz. J. Phys. 48, 190 (2018).

${ }^{3}$ H. Bruus, Lab Chip 12, 1014 (2012).

${ }^{4}$ T. Laurell, F. Petersson, and A. Nilsson, Chem. Soc. Rev. 36, 492 (2007).

${ }^{5}$ A. Ozcelik, J. Rufo, F. Guo, Y. Gu, P. Li, J. Lata, and T. J. Huang, Nat. Methods 15, 1021 (2018).

${ }^{6}$ L. Meng, F. Cai, F. Li, W. Zhou, L. Niu, and H. Zheng, J. Phys. D 52, 273001 (2019).

${ }^{7}$ K. Bücks and H. Müller, Z. Phys. 84, 75 (1933).

${ }^{8}$ H. W. S. Clair, Rev. Sci. Instrum. 12, 250 (1941).

${ }^{9}$ R. R. Whymark, Ultrasonics 13, 251 (1975).

${ }^{10}$ W. J. Xie and B. Wei, Appl. Phys. Lett. 79, 881 (2001).

${ }^{11}$ J. K. R. Weber, C. A. Rey, J. Neuefeind, and C. J. Benmore, Rev. Sci. Instrum. 80, 083904 (2009).

${ }^{12}$ T. G. Wang, M. M. Saffren, and D. D. Elleman, AIAA Paper No. 74-155, 1974.

${ }^{13}$ D. Foresti, M. Nabavi, M. Klingauf, A. Ferrari, and D. Poulikakos, Proc. Natl. Acad. Sci. 110, 12549 (2013).

${ }^{14}$ D. Koyama and K. Nakamura, IEEE Trans. Ultrason. Ferroelectr. Freq. Control 57, 1152 (2010).

${ }^{15}$ D. Koyama and K. Nakamura, IEEE Trans. Ultrason. Ferroelectr. Freq. Control 57, 1434 (2010).

${ }^{16}$ T. Vasileiou, D. Foresti, A. Bayram, D. Poulikakos, and A. Ferrari, Sci. Rep. 6, 20023 (2016).

${ }^{17}$ Y. Ochiai, T. Hoshi, and J. Rekimoto, PLoS One 9, e97590 (2014).

${ }^{18}$ T. Hoshi, Y. Ochiai, and J. Rekimoto, Jpn. J. Appl. Phys., Part 1 53, 07 KE07 (2014).

${ }^{19}$ Y. Ochiai, T. Hoshi, and J. Rekimoto, ACM Trans. Graphics 33, 1 (2014).

${ }^{20}$ R. H. Morris, E. R. Dye, P. Docker, and M. I. Newton, Phys. Fluids 31, 101301 (2019).

${ }^{21}$ J. E. Curtis, B. A. Koss, and D. G. Grier, Opt. Commun. 207, 169 (2002).

${ }^{22}$ K. Dholakia, G. Spalding, and M. MacDonald, Phys. World 15, 31 (2002).

${ }^{23}$ D. G. Grier, Nature 424, 810 (2003).

${ }^{24}$ A. Marzo, S. A. Seah, B. W. Drinkwater, D. R. Sahoo, B. Long, and S. Subramanian, Nat. Commun. 6, 8661 (2015).

${ }^{25}$ S. Inoue, S. Mogami, T. Ichiyama, A. Noda, Y. Makino, and H. Shinoda, J. Acoust. Soc. Am. 145, 328 (2019).

${ }^{26}$ A. Marzo and B. W. Drinkwater, Proc. Natl. Acad. Sci. 116, 84 (2019).

${ }^{27}$ K. Melde, A. G. Mark, T. Qiu, and P. Fischer, Nature 537, 518 (2016).

${ }^{28}$ G. Memoli, M. Caleap, M. Asakawa, D. R. Sahoo, B. W. Drinkwater, and S. Subramanian, Nat. Commun. 8, 14608 (2017).

${ }^{29}$ S. Polychronopoulos and G. Memoli, Sci. Rep. 10, 4254 (2020).

${ }^{30}$ A. Marzo, A. Ghobrial, L. Cox, M. Caleap, A. Croxford, and B. W. Drinkwater, Appl. Phys. Lett. 110, 014102 (2017).

${ }^{31}$ J. Zhang, Y. Tian, Y. Cheng, and X. Liu, Appl. Phys. Lett. 116, 030501 (2020).

${ }^{32}$ S. Zhao and J. Wallaschek, Arch. Appl. Mech. 81, 123 (2011).
${ }^{33}$ M. A. B. Andrade, A. L. Bernassau, and J. C. Adamowski, Appl. Phys. Lett. 109, 044101 (2016).

${ }^{34}$ M. A. B. Andrade, F. T. A. Okina, A. L. Bernassau, and J. C. Adamowski, T. Acoust. Soc. Am. 141, 4148 (2017).

${ }^{35}$ A. Marzo, M. Caleap, and B. W. Drinkwater, Phys. Rev. Lett. 120, 044301 (2018).

${ }^{36}$ M. A. B. Andrade, N. Pérez, and J. C. Adamowski, J. Acoust. Soc. Am. 136, 1518 (2014).

${ }^{37}$ N. Perez, M. A. B. Andrade, R. Canetti, and J. C. Adamowski, J. Appl. Phys. 116, 184903 (2014).

${ }^{38}$ K. Hasegawa and K. Kono, AIP Adv. 9, 035313 (2019).

${ }^{39}$ T. Fushimi, T. L. Hill, A. Marzo, and B. W. Drinkwater, Appl. Phys. Lett. 113, 034102 (2018).

${ }^{40}$ R. Hirayama, D. Martinez Plasencia, N. Masuda, and S. Subramanian, Nature 575, 320 (2019).

${ }^{41}$ M. A. B. Andrade, S. Polychronopoulos, G. Memoli, and A. Marzo, AIP Adv. 9, 035020 (2019).

${ }^{42}$ M. A. B. Andrade and A. Marzo, Phys. Fluids 31, 117101 (2019).

${ }^{43}$ P. Helander, E. Haggstrom, T. Puranen, A. Merilainen, G. Maconi, A. Penttila, M. Gritsevich, I. Kassamakov, A. Salmi, and K. Muinonen, in 2019 IEEE International Ultrasonics Symposium (IEEE, 2019), pp. 650-653.

${ }^{44}$ D. Baresch, J. L. Thomas, and R. Marchiano, Phys. Rev. Lett. 121, 074301 (2018).

${ }^{45}$ L. Zhang and P. L. Marston, Phys. Rev. E 84, 065601 (2011).

${ }^{46}$ M. A. B. Andrade, G. Simon, A. Bernassau, and A. Marzo, in 2019 IEEE International Ultrasonics Symposium (IEEE, 2019), pp. 258-264.

${ }^{47} \mathrm{~K}$. Hasegawa, A. Watanabe, A. Kaneko, and Y. Abe, Micromachines 11, 343 (2020).

${ }^{48}$ L. P. Gor'kov, Sov. Phys. Dokl. 6, 773 (1962)

${ }^{49}$ E. H. Trinh and J. L. Robey, Phys. Fluids 6, 3567 (1994).

${ }^{50}$ K. Hasegawa, Y. Abe, A. Kaneko, Y. Yamamoto, and K. Aoki, Microgravity Sci. Technol. 21, 9 (2009).

${ }^{51}$ K. Hasegawa, L. Qiu, A. Noda, S. Inoue, and H. Shinoda, Appl. Phys. Lett. 111, 064104 (2017).

${ }^{52}$ T. G. Wang and F. H. Busse, J. Acoust. Soc. Am. 69, 1634 (1981).

${ }^{53}$ E. Leung, N. Jacobi, and T. Wang, J. Acoust. Soc. Am. 70, 1762 (1981).

${ }^{54}$ M. A. B. Andrade, T. S. Ramos, F. T. A. Okina, and J. C. Adamowski, Rev. Sci. Instrum. 85, 045125 (2014).

${ }^{55}$ S. Tsujino and T. Tomizaki, Sci. Rep. 6, 25558 (2016).

${ }^{56}$ M. A. B. Andrade, T. S. Ramos, J. C. Adamowski, and A. Marzo, Appl. Phys. Lett. 116, 054104 (2020).

${ }^{57}$ K. Choi, A. H. C. Ng, R. Fobel, and A. R. Wheeler, Annu. Rev. Anal. Chem. 5, 413 (2012).

${ }^{58}$ S. K. Cho, H. Moon, and C. J. Kim, J. Microelectromech. Syst. 12, 70 (2003).

${ }^{59}$ A. Watanabe, K. Hasegawa, and Y. Abe, Sci. Rep. 8, 10221 (2018).

${ }^{60}$ M. A. B. Andrade, T. S. A. Camargo, and A. Marzo, Rev. Sci. Instrum. 89, 125105 (2018)

${ }^{61}$ A. Marzo, A. Barnes, and B. W. Drinkwater, Rev. Sci. Instrum. 88, 085105 (2017).

${ }^{62}$ A. Marzo, T. Corkett, and B. W. Drinkwater, IEEE Trans. Ultrason. Ferroelectr. Freq. Control 65, 102 (2018).

${ }^{63}$ C. J. Benmore and J. K. R. Weber, Phys. Rev. X 1, 011004 (2011).

${ }^{64}$ C. J. Benmore, J. K. R. Weber, A. N. Tailor, B. R. Cherry, J. L. Yarger, Q. Mou, W. Weber, J. Neuefeind, and S. R. Byrn, J. Pharm. Sci. 102, 1290 (2013).

${ }^{65}$ M. Takasaki, D. Terada, Y. Kato, Y. Ishino, and T. Mizuno, Phys. Procedia 3, 1059 (2010).

${ }^{66}$ T. Omirou, A. M. Perez, S. Subramanian, and A. Roudaut, in 2016 IEEE Symposium on 3D User Interfaces (3DUI) (2016), pp. 187-190.

${ }^{67}$ R. Morales, A. Marzo, S. Subramanian, and D. Martínez, in Proceedings of the 32nd ACM Symposium on User Interface Software and Technology, UIST '19 (2019), pp. 651-661.

${ }^{68}$ T. Fushimi, A. Marzo, B. W. Drinkwater, and T. L. Hill, Appl. Phys. Lett. 115, 064101 (2019). 